\title{
Robonaut 2: About New Legs and Technologies
}

\author{
Ms. V. Dhivya \\ Assistant Professor \\ Department of Computer Application \\ Apollo Arts \& Science College, \\ Chennai, India \\ here.dhivya@gmail.com
}

\author{
S. Divagar \\ UG Student \\ Department of Computer Application \\ Apollo Arts \& Science College, \\ Chennai, India \\ divasiva2398@gmail.com
}

\author{
J. Bharath \\ UG Student \\ Department of Computer Application \\ Apollo Arts \& Science College, \\ Chennai, India \\ bharath009j@gmail.com
}

\begin{abstract}
National Aeronautics and Space Administration (NASA) has now a day introducing the Robots to the space for many useful and to do many dangerous technical faults that cannot done by the humans. TheRobonaut 2 which is the dexterous humanoid robot, was designed and introduced by NASA's JSC (Johnson Space Center) in collaboration with General Motors and Oceaneering. The research of the Robonaut 2 that have been started in 1997. And then the research leads to result of R1, human like prototype of a robot that can be mounted on the wheels. And the Space Act Agreement was signed in 2007, for the development of R1.In this paper we discussed about the new legs invention and first movement of R2 in ISS. The technology of the R2 has many benefits and application like Logistics and Distribution, Industrial, Medical and the major work on Hazardous Toxic or Remote Environment. Robonaut 2 is a humanoid with many capabilities allowing it to work side-by-side with humans and on task that would normally not be done by robots.
\end{abstract}

Keywords: Robonaut2, New Legs Invention, First movement on ISS, Industrial field, Medical field.

\section{Introduction}

On February 24, 2011 NASA sends its first humanoid robot to space called Robonaut2 or R2 in its STS133 shuttle mission to the International Space Station (ISS). First,R2 is send to test whether the performance of R2 done on the earth will be affected on space due to loss of gravity and exposure to radiation. It is designed to carry automated tasks or it can be controlled remotely by a human operator.

In Feb 2010, R2- dexterous humanoid robot was introduced. The R2 has many features like Hands, Arms, Sensing and Perception, Interface and Control. And the R2's brain "TORSO". It has a 50 patents, 350 sensors and 42 independent degrees of function. The new R2 climbing legs are suitable for both inside and outside of ISS. Each leg has seven series of elastic joints in an alternating sequence of pitch-roll.Each and every roll joint has a degree of 360range of motion and pitch has a degree of 160 range of motion.

$\mathrm{R} 2$ is checked and inspected based on its processors, sensors; proper functionality, torque measurement, joints and also ensure that there is no damage. These major checkout operations are done with R2 prior it performs space operations.

II. Development of Robonaut
In 1997, First Robonaut was developed to assist the astronauts with another pair of hands. In 2006, Robonaut1 was used to perform various experiments. In 2007,GM and NASA signed agreement to work together on developing Robonaut2.In 2010,February Robonaut2 was unveiled which is faster and more dexterous, more technological advanced humanoid robot then had ever been seen before. In 2011, February24 Robonaut2 was launched and was living in International space Station.

TABLE 1: COST AND SPECIFICATION OF ROBONAUT2

\begin{tabular}{|l|l|}
\hline Basic Tasks & Interact and assist crew \\
Cost & \$2.5 million \\
Materials & Primarily Aluminum with steel \\
Weight & $149.69 \mathrm{~kg}$ \\
Height & 3 feet, 4 inches \\
Shoulder Width & 2 feet,7 inches \\
Sensors & More than 350 \\
Processors & 38 PowerPC processor
\end{tabular}

\section{BODY SEGMENTS OF ROBONAUT2}

A. Head: It has five cameras in that four cameras are used to provide stereo vision for the robot to do its operation.(i.e.) two for stereo vision and two for auxiliary. A fifth camera is mounted in the mouth area for depth perception.

B. Midsection: It contains 38 PowerPC processors controlling R2.

C. Backpack: It holds its power conversion system/batteries.

D. Hands: It has five fingers, which have ability to utilize Internal/External ISS interfaces and tools designed for astronauts. Dexterous fingers have touching sensors and each has grasping force of $2.3 \mathrm{~kg}$

E. Arms: Each arm is about 2 feet, 8 inches and it can hold 20 lbs. Each arm goes seven degree freedom and strength to hold twenty pounds of any pose in any gravity. 


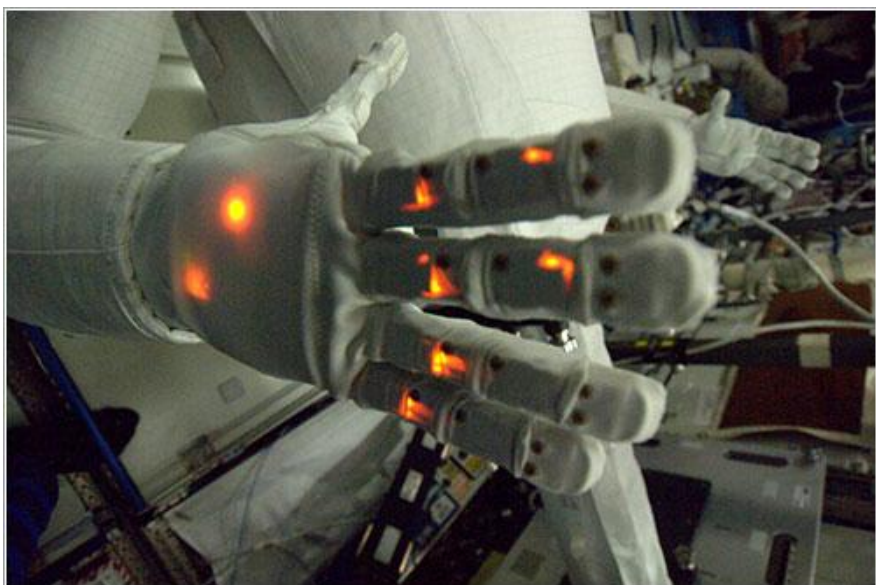

F. Fingers: Robonaut 2 hand has twelve degree of freedom four degree freedom on thumb, three degree freedom of each index and middle finger and one each in ring and pinky finger. Each finger has agrasping force of five pounds.

\section{FIG 1: Fingers Having Touching Sensors}

G. Legs: R2 legs are long and it stretches about 2.7 meters that was exactly 9 feet.

\section{Robonaut2 New Legs Invention}

R2 has a set of legs, it was developed by NASA, set of legs to ISS on September 2014. The legs of R2 were very useful and more flexible to the humans. Because, in space there is a zero gravity, so there is no walking in space, only floating. R2 legs are the functions of grasp footholds, used to move one site to another work site and it can be able to stand gripe in one place to perform a task by its hand.[1]

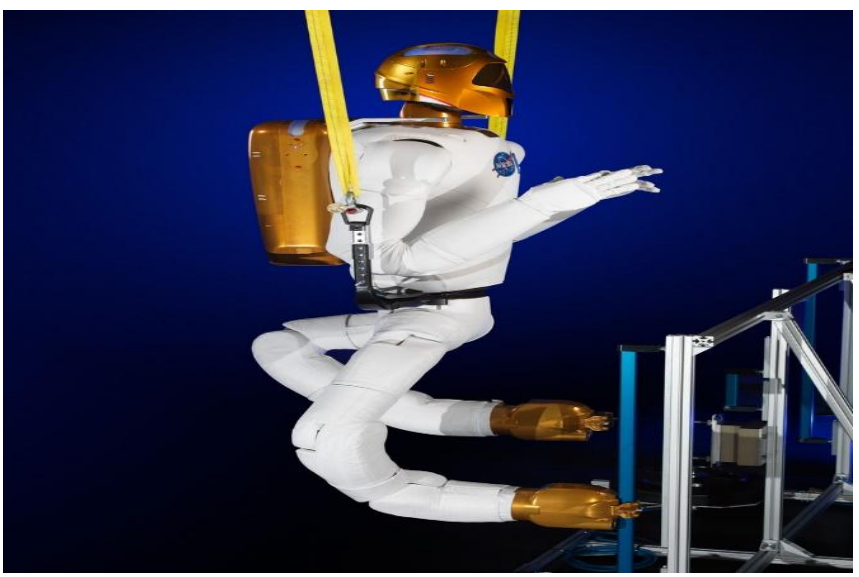

FIG 2: R2 with new Climbing Legs
The new legs were invested by NASA's human exploration and space technology mission directorates which are attached to R2. R2 legs are very long, stretching about 2.7 meters that was exactly 9 feet. When the legs are extended, it can able to done work flexible and it can form wheels like structure. The R2 leg has 7 joints that it can be able to remove and we can attach different parts of space stations.

Since, the new legs are designed to work in a weightless environment. The new legs are not meant for walking; instead these are the climbing legs. It can able to climb everywhere on the space station by using its legs. R2 legs are able to see, by using the cameras on its feet. It has a video camera on its gripper who allows the operator to verify what the robot is doing. The new legs work automatically and it doesn't need any occasional supervision from crew members.

\section{Robonaut2 First Movement On Iss}

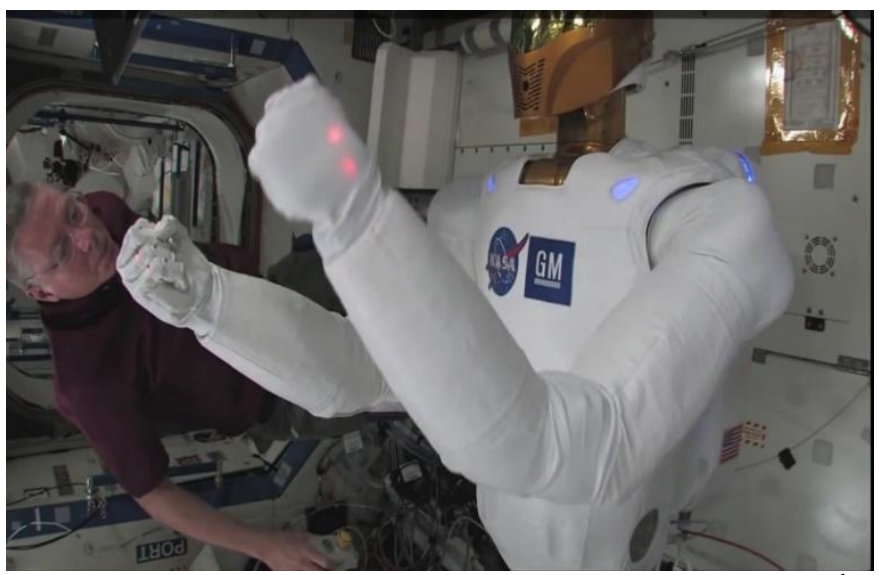

The first movement of R2 on ISS was done on $13^{\text {th }}$ October 2011 with the Astronaut Mike Fossum. The first movement was moving the left arms up and down, then moving hands up and down with 90 degrees and then rotating hands and wrist and so on many more movements. Then tested on right hands.

\section{FIG 3:First movement on ISS with Astronaut Mike Fossum}

\section{Industrial Field}

In order to meet the specifications of the factory floor, R2 was explicitly designed by General Motors. Robonaut is ideally suited for industrial applications. The ability of robots to retool and vary its tasks offers an enormous advantage in a manufacturing environment. In most of the factories, the usages of humans are very less 
percentage because robots are the great part in the industrial field. Many dangerous tasks are not done by humans.

It is possible by robots. And some of the special equipment's and the machines in industrial fields are only controlled by the robots. The equipment and machines that are designed for humans can be operated by R2 such as drills, forklifts. It can turn a gear knob and spin a wheel and also it can fold a piece of fabric and flip a switch. R2 can also be effectively used in the scenarios where dangerous chemicals, biological, or nuclear materials are part of the manufacturing process or in the facility environment.

\section{Medical Field}

R2 technologies can be used in a variety of medical applications, ranging from telemedicine to handling the logistics of medical procedures. Similar to the assembly line on a factory floor, a hospital environment involves repetitive tasks that are ripe for automation. During situations where a biomedical hazards poses risks to humans, such as a contagious outbreak or a combat situations for daily use routine, the technologies of $\mathrm{R} 2$ would be the great advantage for this purpose. It can handle time-consuming tasks of counting, sorting, inspecting, and processing. By handling all these activities by $\mathrm{R} 2$, it frees up hospital staff to focus on their work and it also reduces the human errors.

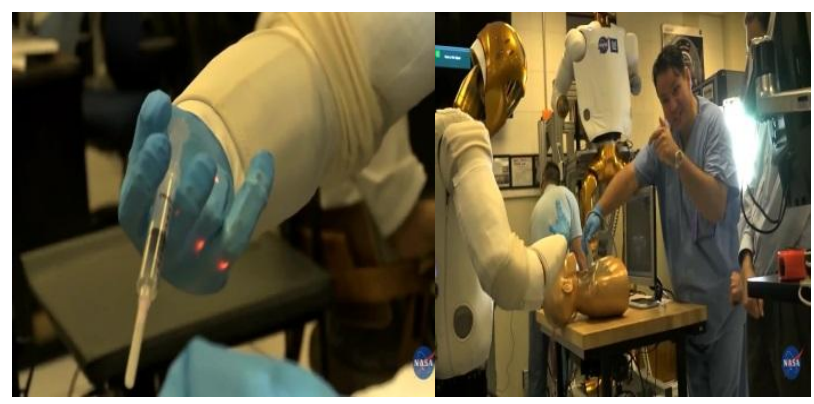

FIG. 4: R2 Experiences in Telemedicine Field

In the field of Telemedicine, now a days Robonaut2are more experienced and robonaut capabilities are demonstrated by experimenting it by its usage of syringe. This demonstration will help to conduct complex medical procedure son humans on many remote locations.

\section{Conclusion and Future Work}

Robonaut2 is designed with human-like structure having 2 arms, a head, 2 legs with end effectors. It is mainly designed to work in a high-risk environment and also it helps on dangerous investigations. Initially R2 was demonstrated by doing duties like removing dust covers and working in task board, once it successfully completes the given task, the additional task will be assigned.

Future enhancement and modifications can be done by allowing it to move more freely throughout the entire stations inside and as well as outside. Also, we can make robonaut teleoperations and humans to work together with robonaut more effectively by using a more advanced algorithms and sensors. [9]

\section{REFERENCES}

[1] Thomas D. Ahlstrom and Andrew W. Curtis "Robonaut 2 on the International Space Station: Status Update and preparation for IVA Mobility"

[2] Stieber. M and Trudel. C and Hunter. D "Robotic systems for the International Space Station," proceedings of the IEEE International Conference on Robotics and Automation, Albuquerque,New Mexico,1997,pp.3068-3073

[3] Hirzinger. G and Brunner. B and Dietrich. J and Heindl. J "ROTEXThe First Remotely Control Robot in Space,"proceedings of the IEEE International Conference on Robotics and Automation, San Diego,California,1994,pp.2604-2611

[4] Oda. M, et al .,"ETS-VII: Achievements, Troubles and Future," proceedings of the $6^{\text {th }}$ International Symposium on Artificial Intelligence and Robotics \& Automation in Space: I-SAIRAS 2001.Montreal,Canada 2001.

[5] Fredrickson .S.E and Lockhart .P.S and Wagenknecht .J.D,"Autonomous Extravehicular Robotic Camera(AERCam) for Remote Viewing,"proceedings of the AIAA International Space Station Service Vehicles Conference,Houston,Texas, 1999.

[6] URL: newatlas.com/robonaut-2-legs/30069

[7] URL: www.rt.com/news/iss-robonaut-new-legs-760

[8] Diftler .M.A., et al., "Robonaut2-The First Humanoid Robot in Space,"proceedings of the IEEE International Conference on Robotics and Automation, Shanghai, China,2011,pp.2178-2183

[9] M.A Diftler and R.Platt "Evolution of the NASA/DARPA Robonaut Control System" 